\title{
La formación transdisciplinaria en el grado universitario: una experiencia de la asignatura de Análisis de la Sustentabilidad Ambiental de Proyectos ${ }^{1}$
}

\section{Enrique Mihura}

Docente investigador de la

Facultad de Ingeniería y Ciencias

Hídricas. Universidad Nacional

del Litoral, Argentina.

enriqueraulmihura@hotmail.com.ar
Sandra Romina Campanella Docente investigadora de la Facultad de Ingeniería y Ciencias Hídricas. Universidad Nacional del Litoral Consejo Nacional de Investigaciones Científicas y Tecnológicas (CONICET). campanellasr@gmail.com

\section{Resumen}

Frente a la crisis ambiental contemporánea es necesario contribuir a la construcción de una "Nueva Sociedad". Para que esto se logre, es necesario generar cambios en los patrones de comportamiento de los sujetos que diariamente conviven en ella. Frente a esta situación y como motores de producción de conocimiento, las universidades no pueden estar excluidas sino que, por el contrario, deben ser un pilar fundamental para lograrlo.

Formar profesionales para un funcionamiento diferente de la sociedad es un gran desafío. Desde esta perspectiva, se trabaja desde la asignatura Análisis de la Sustentabilidad Ambiental de Proyectos de la carrera de Ingeniería Ambiental de la UNL en pos de generar nuevas herramientas para la sociedad actual. La asignatura está incluida como práctica de Extensión Experiencial de la UNL, y dentro de este marco se propone una metodología transdisciplinaria que pretende generar un acercamiento a las problemáticas actuales de la sociedad y buscar las vías y actores más acordes para resolverlas.
Integración de la docencia y la extensión /

Intervenciones

RECEPCIÓN: 12/06/16

ACEPTACIÓN FINAL: 04/08/16

\section{Resumo}

Diante da crise ambiental contemporânea é necessário contribuir para a construção de uma "Nova Sociedade". Para alcançar isto, é necessário gerar mudanças nos padrões de comportamento dos indivíduos que nela convivem diariamente. Perante esta situação e, como motores de produção de conhecimento, as universidades não podem ficar excluídas, pelo contrário, devem ser um pilar fundamental para consegui-lo.

Formar profissionais para um funcionamento diferente da sociedade é um grande desafio. Nesta perspectiva, trabalha-se a partir da disciplina Análise da Sustentabilidade Ambiental de Projetos do curso de Engenharia Ambiental da Universidade Nacional del Litoral (UNL), em prol de gerar novas ferramentas para a sociedade atual. A disciplina está incluída como prática de Extensão Experiencial da UNL e dentro deste marco se propõe uma metodologia transdisciplinar que visa criar uma aproximação dos problemas atuais da sociedade e procurar os meios e os atores mais adequados para resolvê-los.

Palavras-chave

- Transdisciplina

- Sustentabilidade

- Planejar ambientes
- Transdisciplina

- Sustentabilidad

- Proyectar ambientes

\section{Para citación de este artículo}

Mihura, E. y Campanella, S. R. (2016). La formación

transdisciplinaria en el grado universitario: una experiencia de la asignatura de Análisis de la Sustentabilidad Ambiental de Proyectos. En Revista +E versión digital, (6), pp.200-207. Santa Fe, Argentina: Ediciones UNL. 


\section{G6 \\ una metodología con enfoque \\ transdisciplinar implica la interpretación \\ de problemas desde una perspectiva \\ holística, sistémica e integradora}

\section{Introducción}

Desde hace años se habla de una sociedad fragmentada en América Latina (Binder, 1991), donde se puede observar marginación de grupos sociales, beneficios para algunos a costa de otros, grupos aislados que toman decisiones de manera aislada, entre otros. Esto conlleva un sinnúmero de consecuencias que sectorizan a las sociedades por sus diferentes capacidades, formación, etc., relega a algunos sectores y beneficia otros. En el mundo actual, el conocimiento, los sistemas económicos, la organización social, la naturaleza y la realidad se encuentran fragmentados. Esta fragmentación aísla cada componente y partes del ambiente e impide su sustentabilidad (Pesci y otros, 2007). Una posible salida a esta situación es tomar decisiones y generar proyectos que contemplen la complejidad del ambiente de una manera integral, considerando los actores y componentes (naturales, sociales, económicos, etc.) que intervienen. Para lograr esto, es necesario formar sujetos de conocimiento con capacidades y aptitudes para comprender al ambiente desde un enfoque sistémico, como un sistema vivo, abierto y multiescalar, en permanente evolución donde conviven diferentes interacciones. En función de lo mencionado anteriormente, en la asignatura electiva/ optativa Análisis de la Sustentabilidad Ambiental de Proyectos (ASAP) de la carrera de Ingeniería Ambiental de la Universidad Nacional del Litoral (UNL) se trabaja en formar profesionales para un funcionamiento diferente de la sociedad, que puedan aportar conocimientos desde una perspectiva integral. La asignatura está incluida como práctica de Extensión de Educación Experiencial de la UNL, esto permite a los alumnos un "aprender haciendo" y reflexionar sobre "el hacer". El objetivo principal de la asignatura es brindar una herramienta para formar recursos humanos capaces de abordar de manera transversal e integral las problemáticas que existen en la sociedad actual, donde diariamiente desarrollamos nuestras tareas. Dada la metodología que se plantea a lo largo del cursado de la cátedra, implementar prácticas experienciales en el desarrollo de la misma permite fortalecer el vínculo entre "el saber" y "el hacer", y se obtienen así mayor comprensión y apropiación de los alumnos con la temática planteada.

El objetivo de este trabajo es presentar una propuesta de proceso de enseñanza y aprendizaje desde la asignatura ASAP basada en una metodología con enfoque transdisciplinar lo cual implica la interpretación de problemas desde una perspectiva holística, sistémica e integradora. Mediante la misma, el grupo de estudiantes en formación acuerda, en primer término, cuál es el paradigma (criterios, premisas) que rige su accionar y la metodología que vuelve viable el proceso de resolución de los problemas observados/ percibidos con el fin de proyectar ambientes de manera sustentable.

\section{Metodología del proceso enseñanza-aprendizaje}

La metodología propuesta en esta asignatura tiene sus bases en la metodología de "proyectación ambiental" (Pesci y otros, 2007). Esta herramienta puede ser vista como un elemento para lograr una mejor sociedad y promover un trabajo colectivo e inclusivo. Esto incluye una serie de elementos que posibilitan proyectar ambientes al fomentar la conectividad entre el espacio y tiempo y entre los diferentes pensamientos y actores sociales involucrados, unificando intereses para lograr soluciones que beneficien al ambiente en cuestión.

La propuesta original fue adaptada y modificada, transformándose en un proceso de enseñanza-aprendizaje que se encuentra organizado según siete estrategias (Mihura, 2012), no excluyentes. En la Tabla $n^{\circ} 1$ se presentan las estrategias que implementamos en nuestro trabajo.
1) Los autores agradecen a la Secretaría de Extensión, especialmente a la Dirección de Incorporación Curricular de la Extensión de la UNL. Al equipo del Proyecto de Investigación "Procedimientos de Gestión del Desarrollo
Sustentable en Áreas Inundables con Gobernabilidad Difusa" de la Facultad de Ingeniería y Ciencias Hídricas de la UNL; Proyecto de Extensión de Interés Social "Abordaje integral de la tracción a sangre en barrios marginales de
Santa Fe: ampliando acciones" de la Facultad de Ciencias Veterinarias de la UNL y al Proyecto de Extensión de Interés Social "Guardianes del río" de la Facultad de Arquitectura, Diseño y Urbanismo de la UNL. Además, a la
Escuela "Omar Alberto Rupp" de Alto Verde y a la Asociación de Cirujas del barrio Santa Rosa de Santa Fe. 


\section{Estrategia 1}

Tomar conciencia sobre lo que se debería cambiar.

\section{Estrategia 2}

Considerar al ambiente como sistema-proceso de interacciones múltiples y adoptarlo como unidad de desarrollo.

\section{Estrategia 3}

Imaginar y proyectar sociedades que nos permitan lograr y mantener armonía, con los sistemas no construidos por nuestra especie.

\section{Estrategia 4}

Materializar procesos

de desarrollo humano

sustentable.

\section{Estrategia 5}

Cambiar paradigmas en formación de recursos humanos, ciencia y tecnología.

\section{Estrategia 6}

Constituir empresas colectivas microrregionales.

Estrategia 7

"Proyectar ambientes".
Es generar estrategias de educación y reflexión crítica, a partir del concepto de diálogo de saberes, tendientes a aumentar el grado de conciencia existente sobre la creencia/supuesto/paradigma, que "los recursos son finitos y el sistema científico y tec nológico posee limitaciones para restituir, recuperar, reutilizar,

Es entender el ambiente como un sistema/proceso de interacciones múltiples (Mihura, 2010) y considerarlo como el tipo de unidad más adecuado, para la implementación de procesos de desarrollo. Frente a la necesidad de contribuir a revertir las situaciones problemas existentes y con ello de modificar las tendencias a sus agravamientos, se hace necesario llevar la conceptualización de ambiente, a un modelo operativo. Desde la perspectiva explicitada se propone entender al ambiente como un volumen en expansión, de forma indefinida y se plantea que un observador imagine como aproximación a dicha forma, la de una pirámide invertida (Mihura y otros, 1999). Efectuando un corte vertical a la pirámide antes mencionada quedan represen-

Es reflexionar sobre cómo establecer una mejor relación con los sistemas no construidos por nuestra especie. En la dirección señalada se propone iniciar la misma sobre el concepto de sociedad de flujos cíclicos (Pesci y otros, 2007), que plantea una sociedad que encare holística y relacionalmente la realidad,

Es definir el modelo de desarrollo más apropiado y caracterizarlo a partir de concebir el desarrollo como un medio para mejorar la calidad de vida (todas las formas), entendida como la satisfacción de las necesidades humana fundamentales (Max Neef y otros, 1993), en un marco de conservación del patrimonio natural y cultural. Lo expresado consiste en llevar adelante un proceso de crecimiento económico con equidad y

Es enriquecer el sistema de formación de recursos humanos, ciencia y tecnología, debido a que el abordaje de la complejidad y la incertidumbre, que constituyen las características centrales de los conflictos actuales, requieren de una re-contextualización de los abordajes disciplinarios, mediante la progresiva incorporación de los enfoque inter y transdisciplinarios (Pesci y otros, 2007). Dirección en la cual, la evolución del conocimiento, desde la teoría general de sistemas hasta los nuevos desarrollos sobre sistemas complejos o también llamados sistemas vivos efectuados por diferentes escuelas de pensamiento y acción, indican el camino a seguir para efectuarla (Checkland, 1993). En palabras de Gibbons (1998) los conocimientos necesarios están distribuidos y por ende deben ser construidos colaborativamente porque no están todos en la universidad. En este

Es contar con nuevos sistemas de gobernanza, basados en la constitución de asociaciones democráticas entre los sujetos, las organizaciones sociales, los gobiernos y las empresas, que interactúan en cada lugar (Mihura, 1990). La construcción y puesta en funcionamiento del tipo de sistemas mencionado requiere de una conceptualización del poder, que consiste en comprenderlo como la resultante de tres componentes: las relaciones-decisiones, el conocimiento y el capital. A partir

Es entender a los proyectos como procesos (Pesci y otros, 2007): i) de contexto (geográfico, geopolítico, político, social, económico, productivo, etc.); ii) de interacciones múltiples, donde dichas interacciones son fundamentalmente interacciones sociales determinadas por la cultura que las origina; iii) de transformación de la realidad, desde la cultura de la levedad, que buscan cambiar una situación no querida por otra deseada a través de ciclos sucesivos que parten de la observación de un problema $(\mathrm{O})$, continúan con su análisis $(\mathrm{A})$ y gestión de su solución $(G)$ y finalmente verifican el avance alcanzado $(V)$ con relación a la situación deseada; y iv) posibilitadores de diálogo en tiempo y forma, los recursos una vez degradados" y sobre el riesgo que implica, para la continuidad de la vida en el planeta continuar pensando en que "los recursos son infinitos y que su eventual degradación puede ser subsanada por el sistema científico y tecnológico" (Odum, 2012).

tados tantos niveles operativos, de organización y gestión, como lo requiera el trabajo a ejecutar. La identificación de niveles permite visualizar al ambiente como un sistema de coordenadas tiempo espacio, que se asemeja a un campo de fuerzas, con origen tanto en los sistemas no construidos por el hombre (huracanes, sismos, inundaciones, etc.), como en los construidos por éste para su beneficio, como especie. Toda intervención en un sistema/proceso de interacciones múltiples debe ser cuidadosamente diseñada, si el fin que se persigue es proteger y potenciar sus principales atributos. Para ello se requiere de la realización de un diagnóstico complejo y de un creativo y leve proceso de proyectación: indagación, diseño y gestión (Pesci y otros, 2007).

y avanza en el diseño de sus atributos principales desde las siguientes premisas orientadoras: predominio de técnicas integradoras, manejo de todos los tipos de capital, planificación de corto, mediano y largo plazo, ciclos retroactivos y ecosistemas autorrenovables, como unidad productora.

sustentabilidad ambiental (Dourojeanni y otros, 1993) y puede lograr materializarse a través de un proceso de estudio y adaptación (Nuestro Futuro Común, 1987), que solamente requiere contar con capacidad de gobierno (gobernabilidad), s/el ambiente a desarrollar, mediante un sistema de gobierno (gobernanza), que garantice el dialogo de saberes y la concertación estratégica.

camino los ámbitos convencionales donde se lleva a cabo la investigación científica, sobre procesos de desarrollo sustentable deben ser replanteados/ readecuados. Donde los laboratorios para desarrollar experiencias de desarrollo sustentable, deben ser casos que permitan disponer de escenarios reales y en los cuales el sistema de muestreo y análisis debe incluir todos los saberes disponibles. La extensión universitaria en sus múltiples dimensiones es una de las puertas abiertas que la universidad le ofrece a los procesos de cambio en el sentido indicado. Los enfoques y metodologías de investigación deben ser interactivas con el medio y las verificaciones relacionadas con los desarrollos de nuevos sistemas y/o innovaciones en sistemas preexistentes, deben producirse sobre la base de resultados alcanzados en términos de mejorar la calidad de vida.

de lo cual se plantea el diseño de cada unidad sobre la base de una analogía con las grandes empresas.

En este caso la analogía se logra dividiendo el sistema de organización social (red) con asiento en cada unidad micro-regional en tres subsistemas, a saber: Subsistema Político-Institucional (relaciones/decisiones); Subsistema de Ciencia, Tecnología y Formación de Recursos Humanos (el conocimiento); Subsistema Económico (el capital).

de saberes y vehículos de aprendizaje (desde la pedagogía de proyectos). Para proyectar/ implementar un proceso benéfico de transformación, aumentar la resiliencia de un ambiente (sistema-proceso de interacciones múltiples) determinado, el proyectista ambiental debe comprender como está organizado, cuáles son los niveles de gestión que lo componen, cuáles son sus funciones principales, etc. El proyectista de este tipo debe tener una formación transdisciplinaria, que le permita contar con un perfil político-técnico y poseer el estilo Ghandi de hacer política-gerencia de proyectos. (Matus, 1995).

Fuente: elaboración propia 
Teniendo como fundamento teórico estas estrategias, los estudiantes trabajan de manera grupal a lo largo del cursado de la materia. Estos grupos de trabajo son vinculados a proyectos de extensión-investigación de la UNL en donde llevan adelante las prácticas de campo. En este contexto, se da un proceso formativo transdiscplinario que incluye el intercambio de ideas asociadas a una cosmovisión/paradigma ambiental y el pasaje de grupo al equipo. El mismo consiste en proyectar un ambiente determinado siguiendo las etapas que se presentan en la Figura 1. Estas etapas implican analizar el ambiente en cuestión, con sus diferentes actores y componentes, para luego, en función de este análisis, proponer un proyecto o una serie de proyectos que permita atenuar los conflictos más perturbadores que existen y fortalecer las potencialidades.

Es importante mencionar que en la metodología se trabaja fuertemente sobre los conflictos y potencialidades del ambiente en cuestión. El primer concepto hace referencia a acciones o componentes que perturban al sistema en análisis, mientras que el segundo implica componentes o acciones sobre las cuales se puede trabajar para lograr una mejora en el ambiente. El primer nivel de comprensión implica caracterizar el ambiente para luego culminar con la determinación de las premisas/deseabilidades que tiene el equipo frente a éste. El segundo nivel consiste en analizar los conflictos y potencialidades existentes mediante un análisis matricial. De esta manera, es factible identificar el subsistema decisor, que es el conflicto que actúa agravando los otros conflictos y disminuye el poder de las potencialidades existentes. El proyecto que se propone en la estrategia de gestión debe tener como meta trabajar para lograr las deseabilidades propuestas en el 1er nivel y para mejorar las condiciones del subsistema decisor.

Esta metodología, en conjunto con las estrategias, es transmitida en la ASAP. El cursado de la asignatura se lleva adelante mediante una clase semanal de cuatro horas durante la cual, en la mitad del tiempo, se desarrollan los contenidos teóricos del programa analítico por parte de los docentes, con intensa participación de los estudiantes mediante preguntas y reflexiones. El tiempo restante se trabaja en taller, en el que los estudiantes se transforman en protagonistas centrales por medio de la aplicación de las ideas y procedimientos que obtienen de las clases teóricas en sus propios casos. Además de esta reunión semanal, cada grupo de estudiantes lleva adelante recorridos en territorio, relacionándose con integrantes de grupos de investigación y extensión de la UNL que desarrollan las tareas en los lugares seleccionados para la práctica de la asignatura. Las actividades totalizan seis horas semanales en promedio.

\section{Resultados de la experiencia}

Los resultados son analizados en función de lo logrado en el cursado de la asignatura del año 2015. Implementar la metodología expresada en la sección anterior requirió un proceso de organización y gestión por parte del equipo docente que incluyó la conformación de grupos, la selección de ambientes y la materialización de vínculos entre los grupos de estudiantes y los ambientes seleccionados. En esta cohorte se conformaron dos grupos de cinco estudiantes (cada uno compuesto con criterios de diversidad, procedencia, género, formación disciplinaria, etc.), quienes ensayaron sus experiencias y reflexiones en el Paraje La Boca (distrito de la Costa) y en el barrio Santa Rosa (distrito Sudoccidental), ambientes pertenecientes a la capital de la provincia de Santa Fe. Los resultados obtenidos de la aplicación de la metodología propuesta con los estudiantes en el cursado de la asignatura pueden ser analizados desde diferentes aspectos: el producido por los estudiantes, el funcionamiento de la metodología implementada en el grado por parte de los docentes, y los resultados logrados en los alumnos.

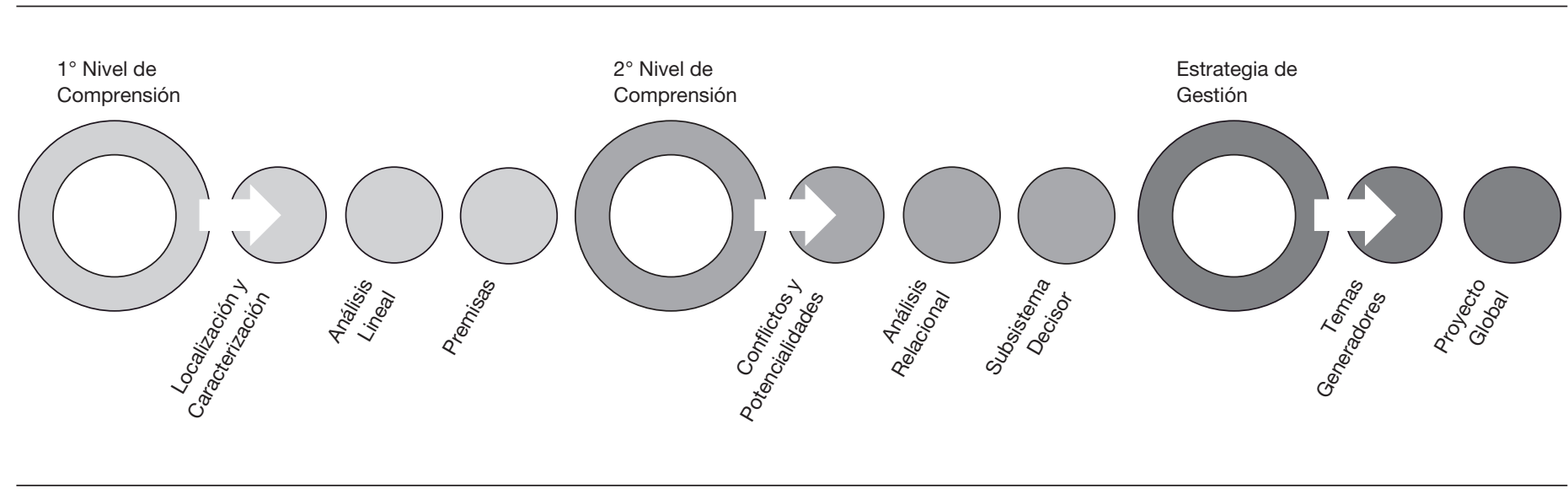

Fuente: elaboración propia sobre la base de Botta y otros, 2015 y Suksdorf y otros, 2015 


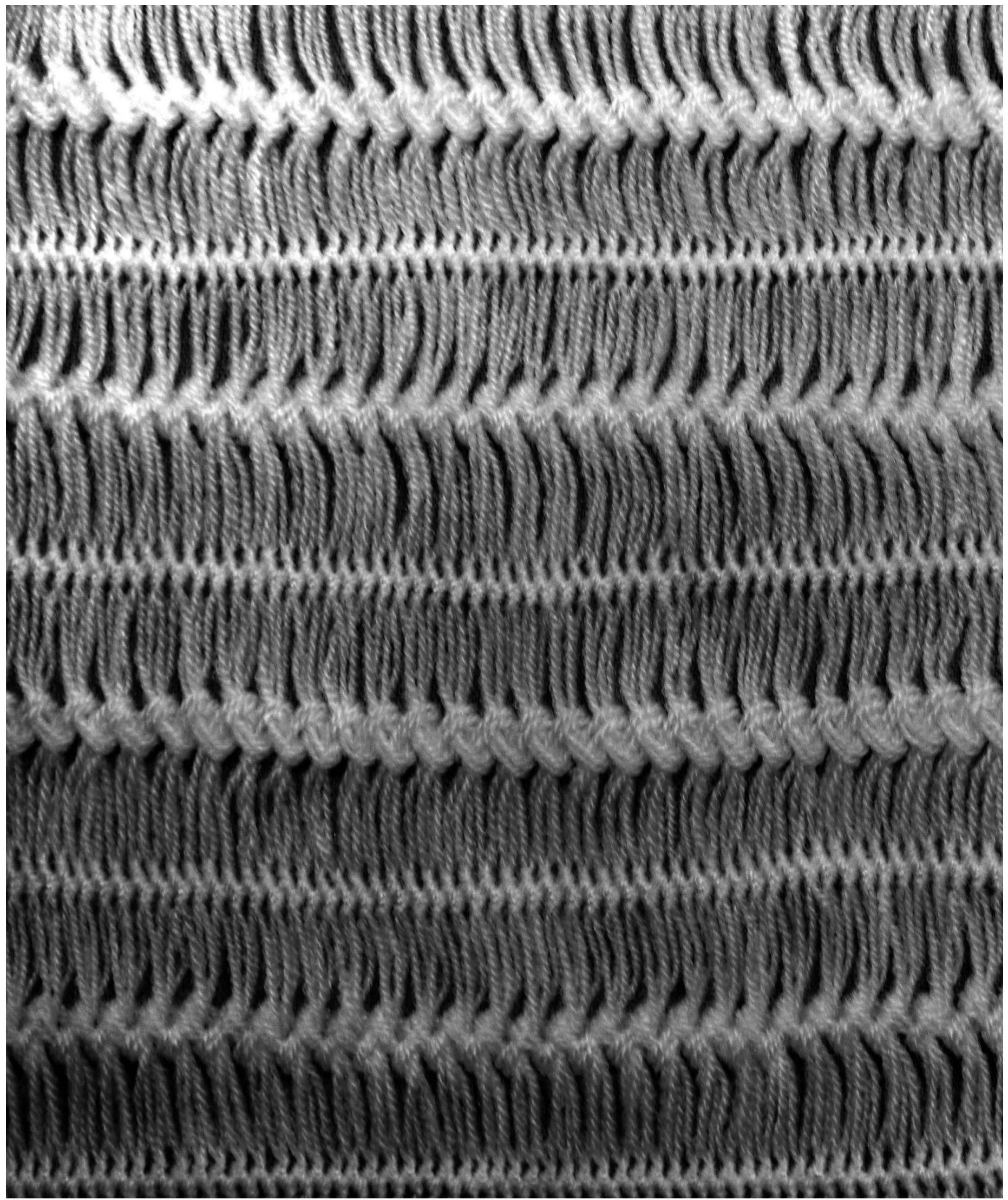


Tabla $n^{\circ} 2$. Resumen de lo producido por los estudiantes

\begin{tabular}{ll}
\hline Grupo & Componentes desarrollados \\
\hline 1. "Caso La Boca" & a) Situación problema: Vulnerabilidad hídrica + Deficiencia de \\
servicios básicos + Condiciones generadoras de enfermeda- \\
des + Imprevisión de servicios sociales estatales + Margina- \\
ción espacial + Falta de propuestas de intervención barrial \\
+ Explotación no sustentable de los recursos + Descontrol \\
del crecimiento territorial y falta de mejora del hábitat + Baja \\
promoción del desarrollo económico. \\
b) Autores del desarrollo involucrados: Estudiantes, equipo \\
docente, equipos docentes asociados, Escuela Rupp, Munici- \\
palidad, Red Interinstitucional Alto Verde. Vecinos de La Boca; \\
UNL; Guardianes del Río; Escuela Rupp; La tranquerita. \\
c) Objetivos de política (deseabilidades): Mejorar la calidad \\
de vida de los habitantes de la Boca en cuanto a condiciones \\
sanitarias que puedan perjudicar el ambiente y su salud. Dis- \\
minuir la vulnerabilidad del lugar frente a las crecidas del río \\
Paraná. Generar mayor acceso a la información y conectividad \\
con el resto de la ciudad de Santa Fe y la micro-región insular \\
que constituye junto con las ciudades de Rincón y Arroyo Leyes. \\
Lograr un desarrollo social del barrio en convivencia y relación \\
sinérgica con el subsistema ecológico. \\
d) Subsistema decisor: Vulnerabilidad hídrica: La Boca se \\
encuentra inmersa en el valle de inundación del río Paraná, \\
por lo que se ve afectado por sus ciclos de crecida. El barrio \\
cuenta con un sistema de defensas sobre el canal de acceso, \\
el cual protege gran parte de las viviendas. Sin embargo, la \\
zona este principalmente, se ve afectada frecuentemente por \\
las crecidas del Colastiné (brazo del Paraná). \\
e) Tema generador: Plan de contingencia enmarcado en el \\
plan global de la ciudad de santa fe contemplando de manera \\
específica las inundaciones del sector este por desborde del \\
río Colastiné.
\end{tabular}

2. "Caso Santa Rosa de Lima" a) Situación problema: Crisis poblacional + Gestión de residuos
inadecuada + Trabajo informal + Uso inadecuado de caballos para trabajo pesado + Exceso de animales en malas condiciones + Deficiencia en infraestructura y Servicios + Deserción escolar + Inacción en materia de seguridad + Escasa inversión en salud y vivienda + Intervención política inadecuada. b) Autores del desarrollo involucrados. Estudiantes, Equipo Docente, Equipos Docentes Asociados, Asociación de Cirujas, Municipalidad, Parroquia. Vecinos de Santa Rosa de Lima y barrios aledaños; Org. Vecinales; Municipalidad de Santa Fe; UNL.

c) Objetivos de política (deseabilidades): Mejorar las condiciones demográficas (relación población-espacio). Mejorar las condiciones actuales respecto a la contaminación de suelos, agua y aire, enfermedades, entre otros. Reducir el grado de vulnerabilidad del área frente a las crecidas de la cuenca del río Salado. Optimizar la gestión de los residuos sólidos. Asegurar el acceso a los servicios básicos en condiciones adecuadas. Minimizar la deserción escolar. Desalentar los procesos de autourbanización y promover procesos de auto construcción regulados por el estado. Aumentar las oportunidades de trabajo formal. Fomentar la implementación de políticas, planes, programas, proyectos y obras, surgidos de la concertación y consenso entre los distintos actores.

d) Subsistema decisor. Crisis poblacional: hace referencia a las malas condiciones de habitabilidad de la población, ya sea por la falta de espacios verdes, terrenos poco estables, la desequilibrada relación población-espacio, los asentamientos precarios y la autourbanización como generadores de la misma. e) Tema generador Talleres de Capacitación Multidisciplinarios "Conociendo mi trabajo": Se pretende aprovechar y mejorar las habilidades asociadas a los diferentes oficios informales que se practican en el barrio, mediante cursos orientados a una capacitación integral por la cual se otorgue algún tipo de certificación.

\section{El trabajo de los estudiantes}

Los estudiantes han podido complementar la teoría y la práctica implementada a lo largo del cursado de manera adecuada y lograr buenos resultados. Llevaron las tareas esperadas durante el cursado de manera organizada, en equipos que involucraban integrantes formados en diferentes disciplinas. De este modo han obtenido informes de buen nivel, que comprenden análisis desde una perspectiva integral y que son un complemento para proyectos de investigación que se están llevando en el área. En la Tabla $n^{\circ} 2$ se expone un resumen del trabajo que llevó adelante cada equipo.

\section{Performance de la metodología implementada}

El proceso de supervisión y evaluación llevado adelante por el equipo docente permitió verificar una muy alta aceptación y una muy buena respuesta de los estudiantes respecto de la metodología que propone la asignatura. Ello quedó expresado en un fluido diálogo de saberes entre los estudiantes, el equipo docente, otros equipos de la universidad y los actores/autores del desarrollo de los ambientes seleccionados, la concertación de intereses alcanzada y plasmada en los proyectos multiactorales formulados y los procesos de gestión asociada que permitieron construirlos y que serán garantía de su implementación.

Asimismo se vio materializado en la colaboración de los estudiantes con el equipo docente, en la preparación y en el dictado del Seminario $\mathrm{V}$ "Estrategias para la construcción de una sociedad sustentable" de la Escuela de Invierno de la UNL (agosto de 2015) y la preparación de los resúmenes de sus experiencias que integran el presente artículo.

En tanto, la metodología empleada quedó demostrada por los procesos académicos en los que los estudiantes se involucraron como expositores en diferentes jornadas ${ }^{2}$ luego de concluir el cursado de la asignatura gracias a los conocimientos adquiridos y a los productos elaborados durante su desarrollo.

\section{Resultados logrados en los estudiantes}

Mediante una encuesta anónima de evaluación docente que se realiza al finalizar el cursado de la asignatura se obtuvieron opiniones por parte de los alumnos. Esta herramienta es de gran valor ya que permite obtener y medir los resultados que se lograron a lo largo del cursado de manera personal, académica y experiencial en cada uno de los estudiantes.

2) Jornadas de Jóvenes Investigadores de la Universidad Nacional del Litoral, 14 y 15 de octubre de 2015, y la Jornada de intercambio y $6^{\circ}$ Taller de
Prácticas de Extensión de Educación Experiencial, 10 y 11 de marzo de 2016, en la Facultad de Humanidades y Ciencias de la UNL. 
En este sentido, se incluye la opinión de cuatro de ellos frente a la pregunta: ¿qué consideras que aprendiste? A continuación realizamos la transcripción de sus opiniones:

"Aprendí a escuchar al otro, más específicamente a saber cuáles son sus necesidades, a trabajar en equipo y a mirar la realidad de otra forma. Pude ver al ambiente como un todo, y logré entender que influyen diversos aspectos en el mismo, muchos de ellos impensados. Esta materia despertó en mí ganas de trabajar y entusiasmo, y forjó en mí la idea de llevar a cabo casi cualquier proyecto que tenga en mente en algún momento de mi carrera profesional. Aprendí que casi siempre las cosas son posibles y que los límites que parecen imponerse no son tan estrictos". (Estudiante 1)

"Aprendí conceptos muy útiles para mi vida, mi carrera y el futuro trabajo que pueda hacer en relación a la construcción de una nueva y mejor sociedad. También pude ver las adversidades que se presentan a la hora de hacer un proyecto, de armar un grupo de trabajo, de realizar las actividades planificadas en tiempo y forma. Por otro lado, pude ver materializado el valor del aporte de cada persona desde su perspectiva y cosmovisión. La materia me ayudó a poder tener una compresión más profunda del tiempo en el que estamos y cuáles son sus rasgos particulares". (Estudiante 2).

"Aprendí una metodología de la clase de ASAP para brindar soluciones mediante desarrollo de proyectos, logrando tener conciencia sobre lo que se debería cambiar. Ser un proyectista consciente de los conflictos y saber qué tipo de acción posible se puede desarrollar ante las situaciones que se presenten en cualquier lugar. De acuerdo con lo que estuvimos viendo en las exposiciones de clases, debemos conformar asociaciones entre los individuos, organizaciones sociales, gobierno y empresas para trabajar colectivamente por un bien común". (Estudiante 3)

"Es algo diferente a lo que venimos acostumbrados en el plan curricular de la carrera. Siendo sincera, me sentí 'incómoda' con la experiencia en el sentido de que constantemente debí lidiar con la difícil tarea de realizar un trabajo desde la subjetividad... Comencé a aprender cómo llevar a cabo un trabajo... Digo que comencé ya que es algo que deberé ejercitar y reaprender de aquí en adelante. Aprendí en determinadas ocasiones a salir de la estructura y ver un poco más allá de lo que se tiene, a 'abrir la cabeza'". (Estudiante 4).

\section{Conclusiones}

El enfoque metodológico propuesto ha demostrado ser una herramienta útil para que los estudiantes puedan tener una mirada colectiva de lo que sucede en un ambiente determinado, de cómo analizarlo, desde qué perspectiva y cuáles son los puntos de partida que permiten llevar adelante un análisis íntegro de la situación problema. Esto, agregado a la posibilidad de realizar prácticas de educación, permite al estudiante conceptualizar la teoría en una aplicación práctica donde el equipo docente puede intervenir y discutir sobre sus percepciones, lo que enriquece al aprendizaje de los estudiantes ya que ellos ponen en práctica una metodología que pueden luego utilizarla en su vida profesional.

Por otro lado, al considerar a los estudiantes simultáneamente como sujetos de aprendizaje y de producción de conocimientos, en la mayoría de los casos, se incrementa su autoestima y compromiso para efectuar un proceso de aprendizaje más consciente y contribuir con ideas y propuestas innovadoras a la solución de los conflictos actuales y con ello al enriquecimiento de los contenidos de la asignatura.

La experiencia concretada deja en evidencia que los períodos cuatrimestrales permiten un desarrollo limitado de los procesos de enseñanza-aprendizaje experienciales. Si bien se alcanza a montar el sistema, a realizar un recorrido completo del flujo metodológico propuesto y generar productos aceptables, las interacciones antes mencionadas no logran la dimensión deseada y los estudiantes no cuentan con el tiempo para efectuar las parte más rica del aprendizaje que consiste en análisis situacionales y diacrónicos del proceso que los tuvo de protagonistas, resignificar, redefinir, ajustar, cambiar, etc. Tareas pendientes que requerirían de un período anual para cumplirse y a través de las cuales el proceso de maduración de los estudiantes sería satisfactorio.

Desde la perspectiva que se plantea la asignatura, se observa que al final del cursado los alumnos desarrollan una visión más integradora de cómo abordar la formulación de proyectos, pueden identificar cuál es el conflicto más perturbador del ambiente a analizar y cuáles serían las mejores soluciones. La metodología demuestra que es factible generar un acercamiento a las problemáticas actuales y buscar las vías y actores más acordes para resolverlas. Con ello también es posible formar sujetos transdisciplinarios que contribuyan a generar un cambio sustentable en la sociedad. 


\section{6 \\ es posible formar sujetos \\ transdisciplinarios que contribuyan \\ a generar un cambio sustentable \\ en la sociedad}

\section{Referencias bibliográficas}

Binder, A. (1991). La sociedad Fragmentada. Caracas. Mimeo

Botta, M.; Benites, I.; Primo, D. y Rubio, X. (2015). Proyectación ambiental, una experiencia en el barrio Santa Rosa de Lima. Encuentros de Jóvenes Invesitgadores UNL. Santa Fe, Argentina.

Comisión Mundial del Medio Ambiente y el Desarrollo (1987). Nuestro Futuro Común. Madrid: Alianza.

Checkland, P. (1993). Pensamiento de Sistemas, Práctica de Sistemas. México: Megabyte.

Dourojeanni, A. (1993). Procedimientos de Gestión para el Desarrollo Sustentable (Aplicados a Municipios, Microrregiones y Cuencas). Comisión Económica para América Latina y el Caribe (CEPAL).

Gibbons, M. (1998). La pertinencia de la educación superior en el siglo XXI. Conferencia Mundial sobre la Educación Superior de la UNESCO. París, Francia.

Matus, C. (1995). Chimpance, Machiavelli y Gandhi. Caracas: Editores Individuales. Mihura, E. R. (1990). Proyecto Final. Curso de Posgrado en Formación Ambiental. Facultad Latinoamericana de Ciencias Ambientales. Auspicio UNESCO. La Plata, Argentina.
Mihura, E. R. (2010). Reflexiones y aportes para la sustentabilidad de procesos de gestión alternativa de la Educación Superior: estrategias para una Educación para el Desarrollo Sustentable. Tesis Maestría en Desarrollo Sustentable. FLACAM-UNLa. La Plata, Argentina.

Mihura, E. R. (2012). Ambiente, Sociedad y Desarrollo: Estrategias de Desarrollo Sustentable. Foro Latinoamericano de Desarrollo Sostenible. Santa Fe, Argentina. Mihura, E. R.; Pagnone, D.; Barbagelatta, P.; Kipen, A. y Schunk, R. (1999). Programa de Desarrollo Sostenible para la Cuenca del Río Gualeguay. Informe final del proyecto de Investigación y Desarrollo. UNER. Entre Ríos, Argentina.

Neef, M.; Elizalde, A. y Hopenhayn, M. (1993). Desarrollo a Escala Humana. Montevideo: Nordan Comunidad.

Odum, H.T. y Odum, E. (2012). O Declínio Próspero: Princípios e Políticas. Petrópolis RJ: Vozes.

Pesci, R.; Perez, J. y Pesci, L. (2007). Proyectar la Sustentabilidad: enfoque y metodología de FLACAM para proyectos de sustentabilidad. CEPA/FLACAM. Suksdorf, F. y Bellomo, F. (2015). Proyectación ambiental, una experiencia en el barrio La Boca Encuentros de Jóvenes Invesitgadores UNL. Santa Fe, Argentina. 